\title{
Mamíferos invasores en Uruguay, historia, perspectivas y consecuencias
}

\author{
Invasive mammals in Uruguay, history, perspectives and consequences
}

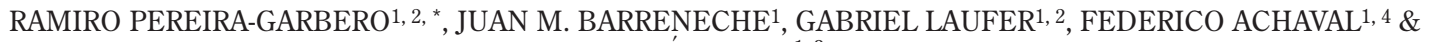 \\ MATÍAS ARIM ${ }^{1,3}$ \\ ${ }^{1}$ Departamento Ecología y Evolución, Facultad de Ciencias, Universidad de la República, Iguá 4225, C.P. 11400, \\ Montevideo, Uruguay \\ 2 Área Biodiversidad y Conservación, Museo Nacional de Historia Natural, Uruguay, 25 de mayo 582 - CC. 399, CP. 11000 \\ ${ }^{3}$ Center for Advanced Studies in Ecology \& Biodiversity (CASEB), Pontificia Universidad Católica de Chile, Santiago, Chile. \\ ${ }^{4}$ In memoriam \\ ${ }^{\star}$ Autor correspondiente: rpereirag@gmail.com
}

\section{RESUMEN}

Relevar la presencia de especies exóticas y conocer sus atributos ecológicos es un importante insumo para la conservación de la biodiversidad. A través de una revisión bibliográfica y de colecciones científicas, se recabaron datos de dieta, tamaño corporal, historia y estatus de invasión de los mamíferos exóticos reportados en Uruguay. Se realizaron modelaciones de nicho para cada especie en base a modelos de Máxima Entropía. También se estimó el cambio generado en la estructura trófica de la biota de mamíferos del Uruguay causado por los procesos de introducción y pérdida de especies. El cambio en los tamaños corporales se evaluó en relación a los datos de distribución de tamaños estimados para los principales grupos tróficos de mamíferos. Este análisis se realizó a nivel de riqueza de especies y biomasa total por grupo trófico, estimada a partir de alometrías conocidas. Los resultados sugieren que el cambio en biomasa es estimable sólo conociendo el exponente de las alometrías entre densidad y tamaño corporal, no requiriéndose conocer la constante de normalización. Las especies recientemente extintas son de mayores tamaños corporales y frecuentemente de dietas carnívoras en comparación a las actuales. Contrariamente, las especies introducidas son omnívoras (Mus musculus, Rattus norvegicus, Rattus rattus, Sus scrofa) y herbívoras (Lepus europaeus, Bubalus bubalis, Capra hircus, Axis axis, Dama dama). Estos cambios en la composición de especies se ven exacerbados cuando los consideramos desde el punto de vista de la biomasa. Asimismo evaluamos la idoneidad climática del territorio uruguayo para el establecimiento de dos especies potencialmente invasoras, la ardilla de vientre rojo (Callosciurus erythraeus) que estaría expandiendo su rango de distribución en Argentina y el visón americano (Neovison vison) que ha sido recientemente introducido con fines de cría comercial. No obstante la relativa homogeneidad geográfica y climática del paisaje de Uruguay, las estimaciones de idoneidad ambiental para el potencial de establecimiento de especies exóticas indican importantes variaciones a lo largo del territorio y entre especies. Nuestro estudio remarca la fortaleza de la teoría ecológica para adelantarse en el estado básico de las especies y su impacto, incluso cuando la información disponible sobre éstas y los sitios de invasión es limitada.

Palabras clave: invasión, mamíferos, modelos de distribución de especies, redes tróficas, tamaño corporal, Uruguay.

\section{ABSTRACT}

It is an important input for conservation of biodiversity to survey the presence of exotic species and to know their environmental attributes. Through literature reviews and scientific collections, we were able to collect data on diet, body size, history and status of invasion of exotic mammals reported in Uruguay. Niche modeling was conducted for each species based on Maximum Entropy models. Also we estimated the changes generated in the trophic structure of the biota of the mammals in Uruguay caused by the processes of species introductions and losses. Changes in mammal's body size were evaluated in relation to the size distribution data for the main estimated trophic groups. This analysis was performed at the species richness level, and the total biomass per trophic group was estimated considering the known allometric relationships. Our results suggest that the change in biomass can be estimated only by knowing the allometric exponent between density and body size, without knowing the normalization constant. The recently extinct species were of greater body size and more carnivorous diets, often compared to the current ones. In contrast, introduced species are omnivorous (Mus musculus, Rattus norvegicus, Rattus rattus, Sus scrofa) and herbivorous (Lepus europaeus, Bubalus bubalis, Capra hircus, Axis axis, Dama dama). Considering trophic level biomass, these changes in species composition are exacerbated. In addition, we evaluate the climatic suitability of the Uruguayan territory for the establishment of two potentially invasive species, the red-bellied squirrel (Callosciurus erythraeus), which is expanding its range in Argentina, and the American mink (Neovison vison) which has been recently introduced in Uruguay for commercial breeding purposes. Despite the relative landscape geographical and climate homogeneity in Uruguay, our estimates for environmental suitability of the potential establishment of exotic species indicate significant changes over the territory and between species. Our study highlights the strength of ecological theory to advance in the basic status of the species, and their impact, even when information on these and the invasion sites are limited.

Key words: body size, food webs, invasion, mammals, species distribution models, Uruguay. 


\section{INTRODUCCIÓN}

Los procesos de aislamiento geográfico y las invasiones biológicas han dado forma a los patrones actuales de diversidad global (Brown \& Sax 2004). El arribo y establecimiento de nuevas especies es un fenómeno natural que forma parte de los procesos de expansión y dispersión (Gotelli 2008). Quizá el ejemplo más conocido sea el Gran Intercambio Biótico Americano, dónde, al conectarse América del Norte y del Sur mediante el Istmo de Panamá, ocurrió un importante flujo de especies (Marquet \& Cofré 1999, Webb 2006). Sin embargo, la mayoría de los cambios en la biota a lo largo de la historia geológica involucraron un número limitado de especies (May et al. 1995). El arribo de una nueva especie puede tener grandes impactos en la biodiversidad local, y la estructura y funcionamiento de las comunidades nativas (Chapin et al. 2000, O'Dowd et al. 2003). Un caso paradigmático en este sentido, es la especie humana, la cual ha alterado los ecosistemas a lo largo de su expansión y desarrollo, siendo esta modificación, la primera causa de pérdida de biodiversidad a nivel mundial (Pimm et al. 1995, Vitousek et al. 1997, D'Antonio et al. 2001). El traslado de especies con el flujo de personas y mercancías, está produciendo un incremento sin precedentes en el número de eventos y cantidad de individuos involucrados en los procesos de invasión (Jaksic 1998, Ricciardi 2007, Westphal et al. 2008, Wilson et al. 2009, Hulme 2009). Además, las alteraciones provocadas por el hombre pueden incrementar la disponibilidad de recursos para las especies invasoras, o la vulnerabilidad de las comunidades por causa de la degradación del ambiente (Didham et al. 2007, Lockwood et al. 2007, Neill \& Arim 2011). Las interacciones entre especies también pueden afectar los procesos de invasión mediante facilitación (Altieri et al. 2010) y degradación ambiental como consecuencia de invasiones previas (Simberloff \& Von Holle 1999, Relva et al. 2009).

Las invasiones biológicas son un componente central del cambio global, que al combinarse con los procesos de extinciones locales están generando homogenización de biotas a nivel global (D'Antonio et al. 2001, Pyke et al. 2008). Estos cambios en diversidad también implican cambios en la estructura y funcionamiento de las comunidades, relativamente menos estudiados. Considerando la identidad de las especies, las invasoras suelen presentar atributos biológicos diferentes al de las biotas nativas e incluso al de las especies nativas extintas (Mack et al. 2000). En este sentido, mientras la mayoría de las extinciones se darían entre especies de gran tamaño y niveles tróficos superiores, las invasiones tienden a involucrar a especies de niveles tróficos inferiores (Byrnes et al. 2007). Esto produce un cambio en la estructura de las redes tróficas, ampliando su base y reduciendo su cúspide (Byrnes et al. 2007). Estos cambios pueden verse magnificados o atenuados por la identidad de las especies involucradas. Así, los carnívoros invasores han mostrado tener efectos mayores sobre la comunidad de presas que las especies nativas (O'Dowd et al. 2003, Salo et al. 2007), mientras que los herbívoros podrían tener un efecto dispersor, por ejemplo con endozoocoria (Davis et al. 2010) e incluso controlar invasiones vegetales por efecto de antagonismo entre invasores (La Pierre et al. 2010). Además, pueden generarse relaciones de tolerancia o resistencia entre plantas y herbívoros (Bailey \& Schweitzer 2009), como también la sustitución de aquellas que son más palatables por otras menos preferidas (O'ReillyWapstra \& Cowan 2009).

Un grupo en el que se ha reportado este patrón de extinción de depredadores nativos y aumento en herbívoros invasores es el de los mamíferos. Estos representan un componente importante de la mayoría de las comunidades, determinando el flujo de nutrientes (Rooney et al. 2008, Hopcraft et al. 2009), los patrones de depredación (Sinclair et al. 2003), la composición de la estructura vegetal (Croll et al. 2005) y el ambiente físico (Jones et al. 1994, Wright et al. 2002, Wallem et al. 2007). A nivel ecosistémico, la introducción de mamíferos puede afectar el funcionamiento de las redes tróficas, generando efectos en cascada en la composición y abundancia de especies de depredadores, herbívoros y plantas (Croll et al. 2005, Hopcraft et al. 2009) y los ciclos de nutrientes (Wardle et al. 2007). Es así que, los mamíferos herbívoros introducidos pueden actuar como dispersores de semillas, reducir la abundancia o eliminar especies nativas y facilitar o frenar nuevas invasiones (Nuñez et al. 2008, Davis et al. 2010). El 
aumento de herbívoros exóticos puede también producir un aumento en la disponibilidad de presas y la oferta alimentaria para los depredadores nativos (Carlsson et al. 2009). En ciertos casos también se ha reportado que mamíferos introducidos pueden operar como especies ingenieras (Hastings et al. 2007), como sería el caso en de las modificaciones de los sistemas hídricos causadas por el castor, Castor canadensis Kuhl, 1820 en el Sur de Chile y Argentina (Arismendi et al. 2008, Anderson et al. 2009), o la modificación de la fisonomía del paisaje por el ganado vacuno, Bos taurus Linnaeus, 1758 (Naveh et al. 2001).

Sudamérica aparece como una región particular en cuanto a los mamíferos exóticos, contando con el $20 \%$ de las introducciones a nivel mundial (Novillo \& Ojeda, 2008). No obstante, el conocimiento del estado de estas invasiones es fragmentario, restringido a ciertos países (Jaksic \& Yáñez 1983, Bonino \& Soriguer 2004, Guichón et al. 2005, Shepherd \& Ditgen 2005, Scheibler \& Christoff 2007, Baldini et al. 2008, Galetti et al 2009, Pérez Carusi et al. 2009, Rocha et al 2011) y escasamente representado en la bibliografía. De hecho, pocos países de la región tienen un inventario claro de las especies de mamíferos exóticos presentes y menos aún del estado de sus invasiones y de sus impactos. El estado de conocimiento es mayor para el cono sur de Sudamérica donde se reportan 18 mamíferos introducidos para Argentina (Novillo \& Ojeda 2008) y 15 para Chile (Jaksic 1998) y donde existe información de los efectos de estas especies e incluso análisis del frente de dispersión y similitud entre las regiones patagónicas de ambos países (Jaksic et al. 2002). Es fundamental ampliar estos estudios a otros países y evaluar los impactos en la estructura de las comunidades de las especies exóticas y sus interacciones con la fauna nativa, así como el estatus actual o potencial de sus poblaciones (Iriarte et al. 2005, Quiroz et al. 2009).

Este trabajo recopila la información disponible sobre los mamíferos exóticos presentes en Uruguay. En base a esta evidencia se generan hipótesis sobre la idoneidad ambiental de estas especies a lo largo del territorio y por último se emplea un enfoque novedoso para estudiar las invasiones biológicas y su efecto estimando la magnitud de los cambios en la estructura trófica debidos a los procesos de extinción e invasión que están teniendo lugar en la región.

\section{MÉTODOS}

\section{Especies consideradas}

Se recopilaron datos de las especies de mamíferos exóticos que presentan poblaciones en estado silvestre en el país y sus principales atributos biológicos. Los datos de distribución nacional, hábitos tróficos y atributos biológicos, se obtuvieron de reportes de ocurrencia de especies exóticas, listados de fauna y guías de campo nacionales (Figueira 1894, Sanborn 1929, Tálice 1969, Vaz Ferreira 1969, Ximénez et al. 1972, Del Pino 1988, González 2001, Achaval et al. 2007), datos de las colecciones zoológicas del Museo de Historia Natural (MNHN) y de la Colección de Zoología de Vertebrados de Facultad de Ciencias (ZVCM). Para obtener datos de distribución global, se realizaron búsquedas en bases de datos internacionales de libre acceso (Museum de Oklahoma, GBIF (Global Biodiversity Information Facility); GISD (Global Invasive Species Database, IUCN/SSC), IABIN (Inter-American Biodiversity Information Network)) y en fuentes de bibliografía gris o de divulgación e informes técnicos (Loudon \& Curlewis 1988, Raman 1997, Schmitt et al. 1997, Ramakrishnan et al. 1999, Alvarez-Romero et al. 2008, Novillo \& Ojeda 2008). A partir de la información obtenida, se reconstruyó, para cada especie exótica, la historia de invasión, las vías de ingreso, los efectos ecológicos, su manejo por las autoridades nacionales (i.e. requerimientos de permiso de caza Decreto 164/96) y el estado de conservación de sus poblaciones en la distribución original (IUCN 2010). Debido a la escasa información existente, para algunas especies la reconstrucción es fragmentaria o incompleta.

A partir de los registros obtenidos en bibliografía y colecciones, se confeccionó una base de registros independientes georreferenciados para cada especie. Únicamente se consideraron especies con poblaciones establecidas, persistiendo en estado silvestre sin ayuda directa del hombre. Nótese que la modelación de nicho requiere asociar confiablemente las ocurrencias con el ambiente. Esto excluye animales domésticos como perros, gatos y ganado.

\section{Modelación de idoneidad climática}

Los métodos de modelación de distribución de especies se han convertido en una popular herramienta en ecología y conservación (Franklin 2010). Estos métodos permiten estimar el rango final de distribución y evaluar la vulnerabilidad para el establecimiento de especies invasoras (Peterson \& Robins 2003, Ficetola et al. 2007). A partir de la base de datos de ocurrencias obtenida, se realizó la modelación de idoneidad climática para los mamíferos exóticos, generándose mapas de distribución potencial para Uruguay, en base al criterio de máxima entropía, Maxent (ver Phillips et al. 2004',

1 PHILLIPS SJ, M DUDIK \& RE SCHAPIRE (2004) A maximum entropy approach to species distribution modeling. Proceedings of the 21st International Conference on Machine Learning, Julio 2004, ACM Press, New York :655-66 
Phillips \& Dudik 2008, Elith \& Leathwick 2009). Este método presenta la ventaja de utilizar solamente datos de presencia y ha demostrado ser superior a otras alternativas bajo un amplio rango de circunstancias (Phillips et al. 2006, Elith \& Graham 2009, Franklin 2010, Mateo et al. 2011).

Para generar los mapas de distribución potencial, los puntos de presencia de las especies fueron recolectados de las fuentes antes mencionadas, descartándose registros de ubicaciones replicadas. Cuando las coordenadas explícitas no estaban incluidas y el número total de registros era inferior a 30 se utilizaron las coordenadas aproximadas, en base a las localidades reportadas. Esta aproximación ha mostrado tener poca influencia en el resultado final de Maxent (Graham et al. 2008, Fernández et al. 2009). Los datos de las variables ambientales se obtuvieron de las capas ambientales 'bioclim' del sitio web WorldClim (Hijmans et al. 2005), con resolución de 30 segundos de arco $\left(\sim 1 \mathrm{~km}^{2}\right.$ por celda). Los datos de pseudo-ausencias necesarios para correr el algoritmo Maxent fueron obtenidos de estas capas a través de la generación de números aleatorios, utilizando el SIG software GRASS (Grass Development Team 2008 http://grass.osgeo. org). Aparte de descartar puntos ubicados en océanos u otros cuerpos de agua, no se aplicó ninguna restricción a las pseudo-ausencias, obteniéndose un total de 2901 muestras. Con esta metodología se estimó el mapa de idoneidad ambiental para cada una de las especies de mamíferos consideradas. Los mapas obtenidos para cada especie fueron superpuestos utilizando el programa QGis (http://www.qgis.org) para determinar las regiones del Uruguay más propicias para el establecimiento de mamíferos exóticos. No se estimó la idoneidad climática para la vizcacha (Lagostomus maximus Desmarest, 1817) y el conejo (Oryctolagus cuniculus Linnaeus, 1758), ya que la primera fue introducida y erradicada a comienzos del siglo XX y el segundo no cuenta con poblaciones en estado silvestre fuera de las Islas costeras (Tálice 1969, Vaz Ferreira 1969, Del Pino 1988).

\section{Cambios en estructura trófica}

Siguiendo a Byrnes et al (2007) se estimó el cambio en riqueza de especies para los distintos grupos tróficos, herbívoros, omnívoros, insectívoros y carnívoros, de acuerdo a la información disponible. Para el análisis se registraron las especies extintas en tiempos históricos y las consideradas en peligro actualmente (González 2001, Achaval et al. 2007) para cada uno de los grupos tróficos. Se consideraron dos escenarios de cambio en la estructura trófica de la biota de mamíferos. Primero, estimando el cambio en diversidad en cada grupo trófico al incluir las especies introducidas y sustraer aquellas ya extintas. En segundo lugar, se consideró la eventual desaparición de las especies con algún grado de amenaza de conservación, aumentando así el número de sustracciones (González 2001, IUCN 2010).

Los cambios en biomasa por grupo trófico se estimaron en base a alometrías de densidad-tamaño corporal (Damuth 1981, Carbone \& Gittleman 2002, Carbone et al. 2007). A partir de estas relaciones se estimó el cambio esperado en densidad en base a los tamaños corporales de las especies incorporadas y perdidas del pool regional (Damuth 1981, Carbone \& Gittleman 2002, Carbone et al. 2007). La densidad estimada de cada especie (N) se multiplicó por su tamaño corporal (M), obteniendo la biomasa poblacional:

Densidad $(\mathrm{N})=\mathrm{aM}^{\mathrm{b}}$
Biomasa $=\mathrm{N} \times$ Masa corporal $(\mathrm{M})=\mathrm{aM}^{\mathrm{b}} \times \mathrm{M}=\mathrm{aM}^{\mathrm{b}+1}$

La biomasa de cada grupo trófico se estimó sumando la biomasa estimada para cada una de las especies que lo componen:

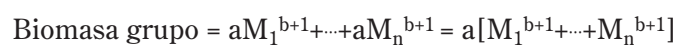

Finalmente, el cambio en biomasa se estimó como la relación entre la biomasa estimada para la fauna actual en relación a la biomasa estimada para la fauna original sin extinción ni incorporación de especies:

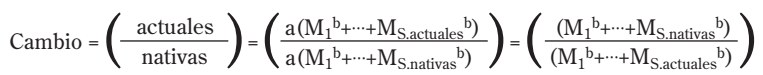

Algunos puntos de esta relación deben ser resaltados. En primer lugar la estimación del cambio en biomasa por grupo trófico es sensible al exponente alométrico pero no al intercepto. Esto es particularmente importante dado que el exponente se conoce aceptablemente bien, pero el intercepto es sensible al sistema de estudio (Brown et al. 2004, Marquet et al. 2004). Así mismo, la estimación del cambio en biomasa es ahora independiente al área, dado que los cambios en área afectan proporcionalmente a todas las densidades afectando sólo al intercepto de la relación.

\section{RESULTADOS}

\section{Mamíferos exóticos en Uruguay}

A partir de la información recabada, se obtuvo la lista de mamíferos invasores para Uruguay, incluyendo sus principales atributos biológicos, los datos existentes sobre el proceso de invasión y su estatus legal (Tabla 1). Al menos 13 especies de mamíferos exóticos han sido introducidas o podrían arribar inminentemente al Uruguay, de las cuales tres no tienen actualmente poblaciones en estado silvestre. Una de estas especies, la vizcacha ( $L$. maximus), fue introducida y ya erradicada, y dos de ellas, la ardilla de vientre rojo (Callosciurus erythraeus Pallas, 1779) y el visón americano (Neovison vison Schreber, 1777) presentan potencial de ingresar al país por cercanía o por introducirse con fines productivos. Si bien la mayor parte de las especies fueron introducidas antes de la primer mitad del siglo XX, para caza y como "hitchhikers", existen algunas introducciones recientes con finalidad productiva ( $N$. vison). Las especies introducidas presentan atributos ecológicos, tamaños corporales y vías de introducción diversas, así como categorías de manejo diferentes por parte de las autoridades nacionales (Tabla 1$)$. 


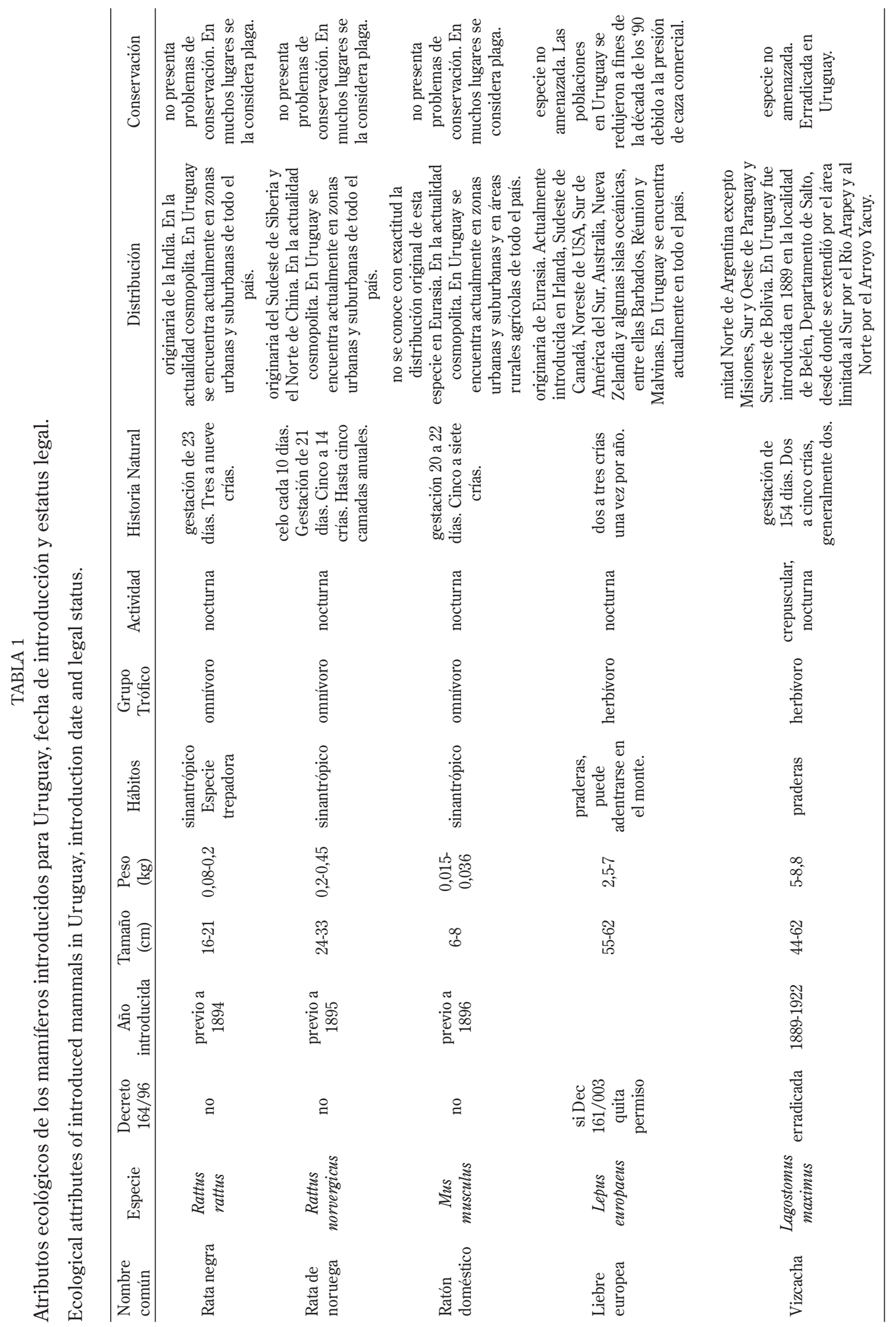




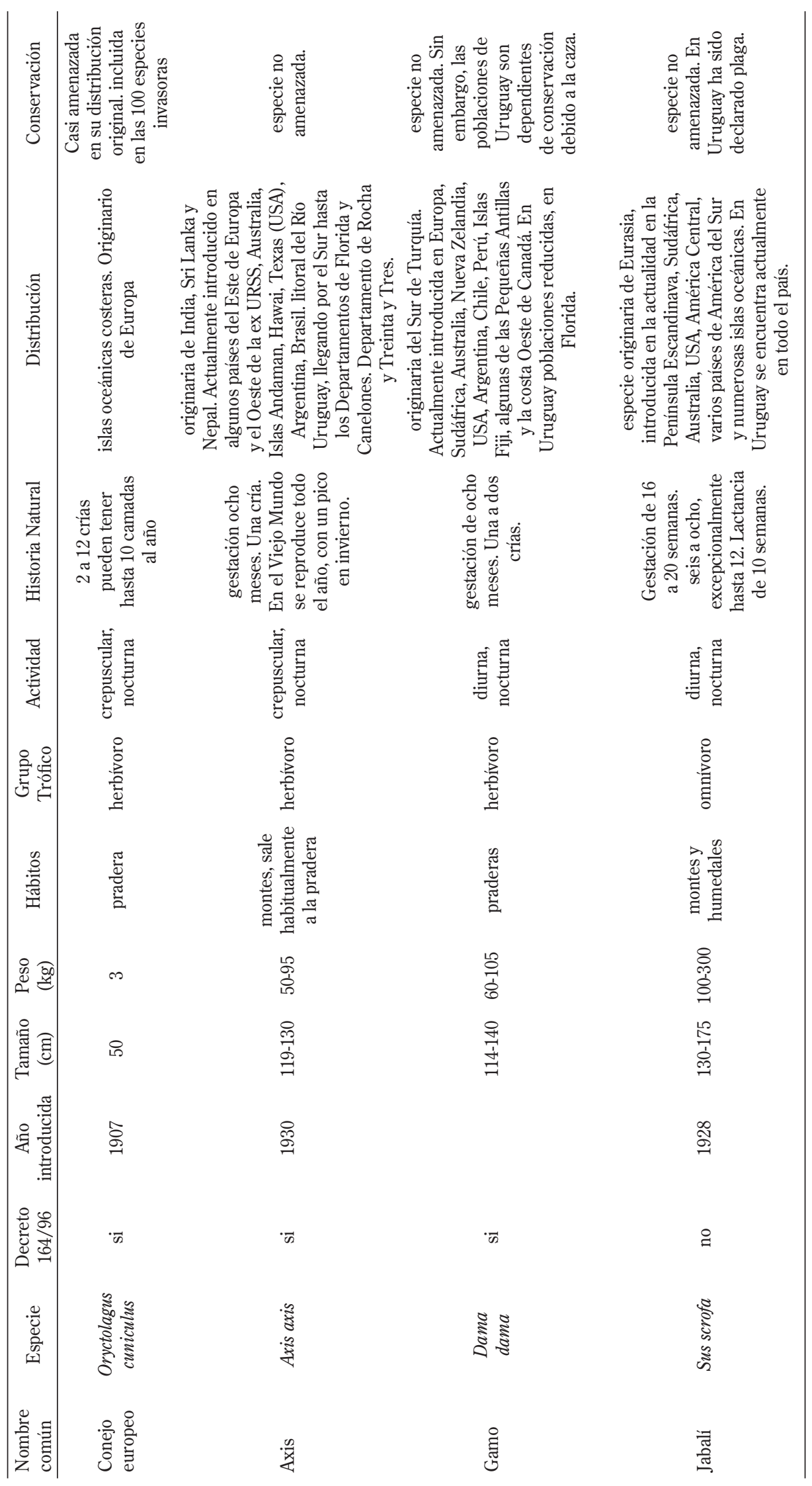




\begin{tabular}{|c|c|c|c|}
\hline 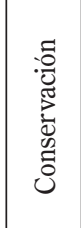 & 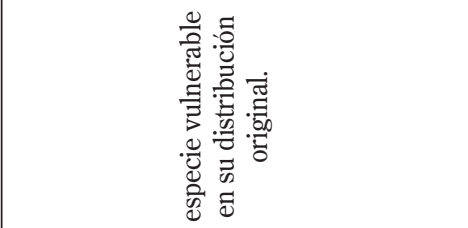 & 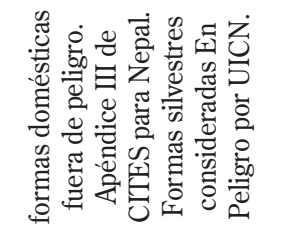 & 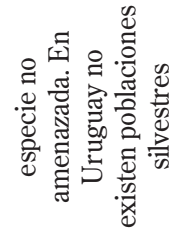 \\
\hline 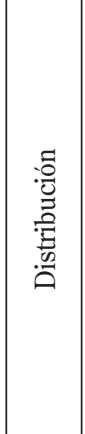 & 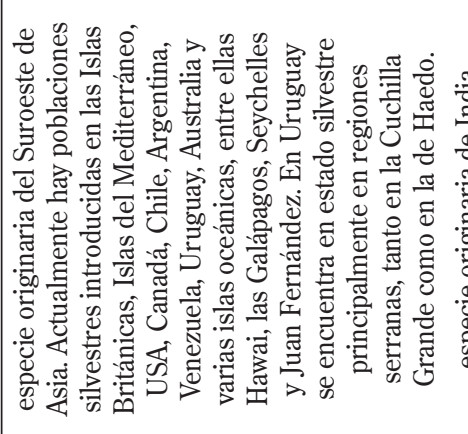 & 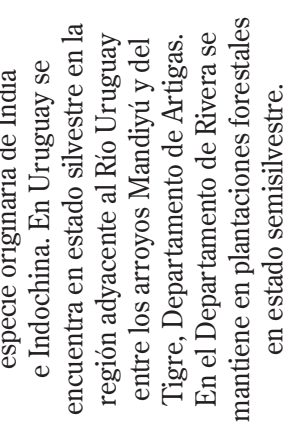 & 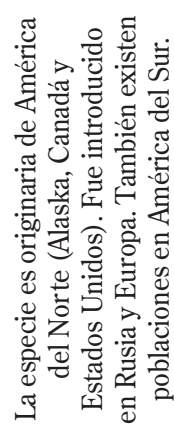 \\
\hline 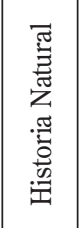 & 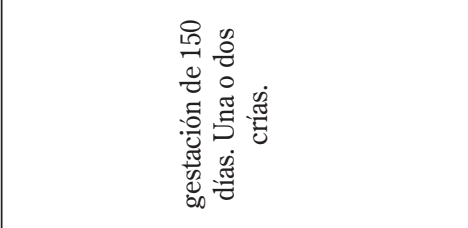 & 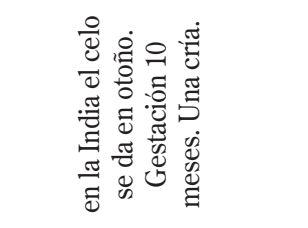 & 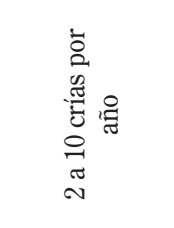 \\
\hline 宽 & 营 & 莺 & 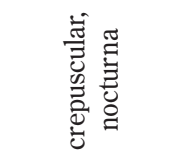 \\
\hline 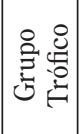 & 竞 & 营 & 冚 \\
\hline 总 & 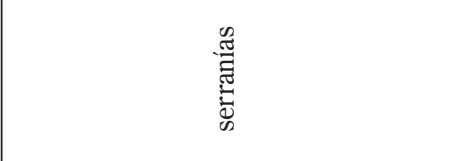 & 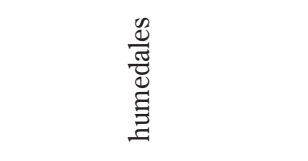 & 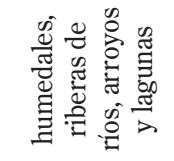 \\
\hline 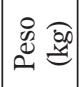 & \& & 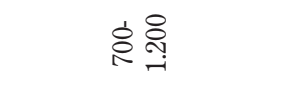 & 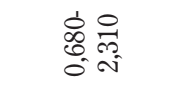 \\
\hline 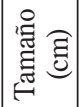 & 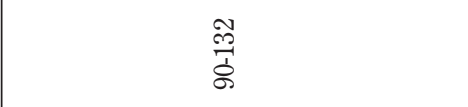 & $\begin{array}{l}\text { तิ } \\
\text { ते̀ }\end{array}$ & 祢 \\
\hline 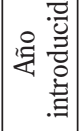 & & & $\stackrel{\infty}{\stackrel{\leftrightarrow}{\circ}}$ \\
\hline 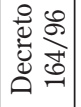 & $\vec{\omega}$ & $\bar{\omega}$ & \\
\hline 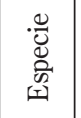 & $\begin{array}{l}\text { s. } \\
\text { s. } \\
\text { s. }\end{array}$ & 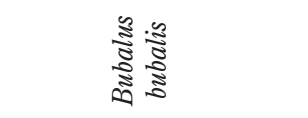 & 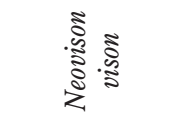 \\
\hline 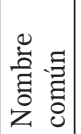 & 苞 & 承兽 & 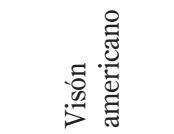 \\
\hline
\end{tabular}



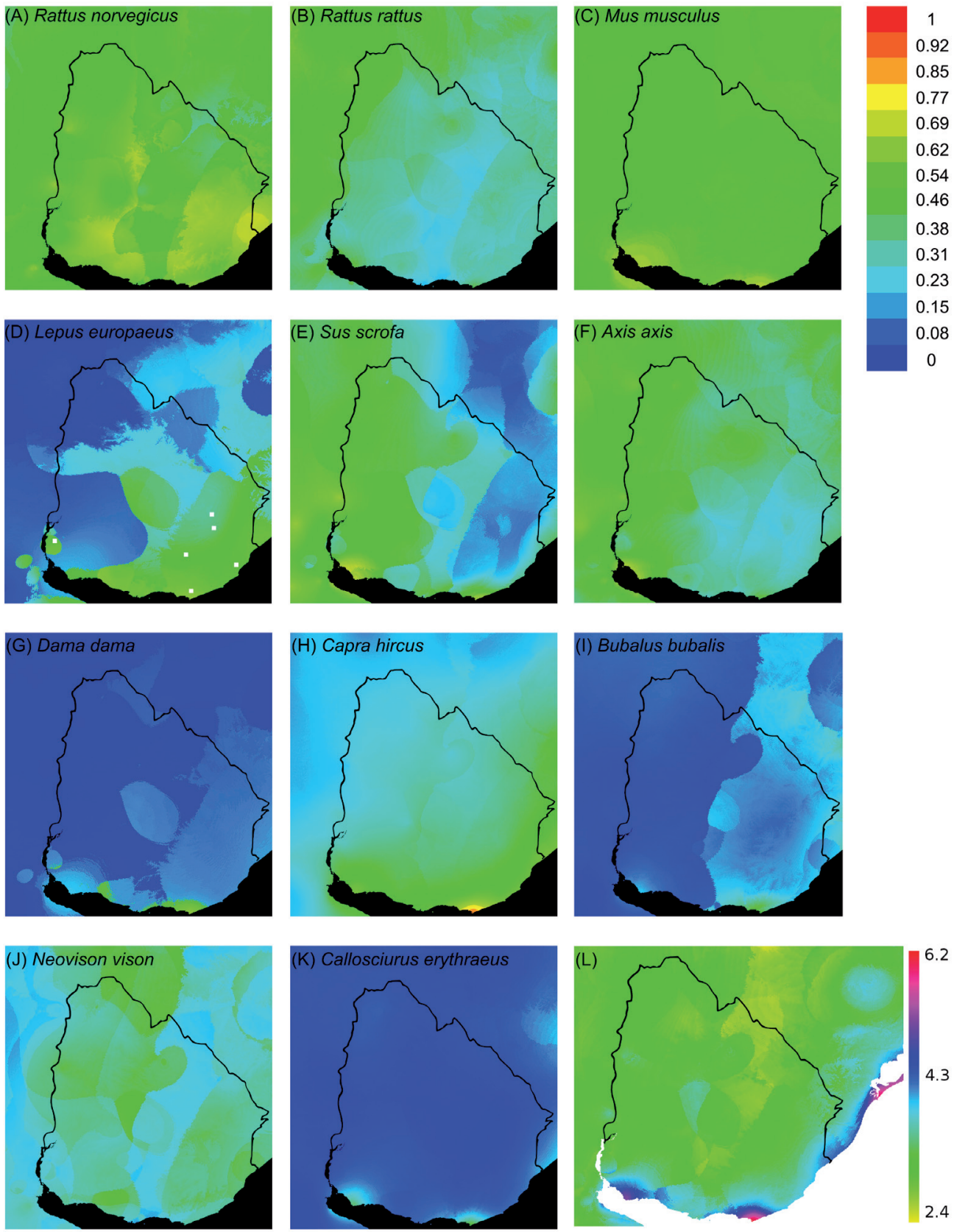

Fig. 1: (A-K) Mapa de idoneidad climática para las especies exóticas actual o potencialmente presentes en el Uruguay. Las predicciones son esencialmente congruentes con los procesos de invasión, estimándose baja idoneidad para aquellas especies con baja dispersión desde su introducción y alta idoneidad para las especies ya presentes en todo el territorio. Se destaca la predicción de alta variabilidad en la idoneidad para especies como el jabalí (Sus scrofa). (L) Mapa de idoneidad acumulado para todas las especies evaluadas. Se destacan dos regiones al sur del país especialmente propicias para el establecimiento de especies exóticas.

(A-K) Maps of environmental suitability for non-indigenous species actual or potentially present in Uruguay. Predictions are essentially congruent with the observed processes of species invasions, with low suitability in those cases with restricted dispersion and high suitability for species actually present in most of the country. It should be highlighted the prediction of high environmental suitability for some species as the feral hog (Sus scrofa). (L) Cummulative suitability map for all species tested. It highlights two regions south of the country especially favorable for the establishment of alien species. 
En general, las especies presentaron patrones de idoneidad de hábitat no homogéneos, implicando la existencia de regiones del Uruguay en donde la posibilidad de establecimiento de especies exóticas es considerablemente mayor (Fig. 1). A continuación se presentan las historias de invasión, los reportes disponibles y las estimaciones obtenidas por Maxent para este conjunto de especies.

Ratas y ratón del viejo mundo (Rattus rattus Linnaeus, 1758, R. norvergicus Berkenhout, 1769, Mus musculus Linnaeus, 1758)

Si bien se desconoce la fecha de ingreso al país de estas especies, las primeras listas de especies del finales del 1800 para el Uruguay, ya las incluyen (Arechavaleta 1882, Aplin 1894, Figueira 1894, Araújo 1900, Sanborn 1929, Devincenzi 1935). Son particularmente comunes en ambientes urbanos y periurbanos en todo el territorio (González 2001, Achaval et al. 2007), incluyendo las islas oceánicas (Vaz Ferreira 1950, Ximénez \& Langguth 2008). Congruentemente, las estimaciones realizadas por Maxent sugieren una probabilidad de éxito relativamente homogéneo para todo el país (Figs. 1A, 1B y 1C). No se conoce mucho de sus interacciones tróficas con la fauna nativa. Las tres especies son depredadas por la lechuza de campanario (Tyto alba Scopoli, 1769), siendo $M$. musculus uno de los ítems más importantes para estas aves en ambientes urbanos (Claramunt \& González 1999²). Estos roedores resultan de interés por los perjuicios económicos que producen y por su relación con la salud humana, son reservorios de varios patógenos incluyendo el causante de la leptospirosis (Zamora \& Riedemann 1999, Conti Díaz 2001).

Liebre europea (Lepus europaeus Pallas, 1778)

Esta especie tiene una amplia distribución en la región Sur de Sudamérica, encontrándose en

2 CLARAMUNT S \& EM GONZÁLEZ (1999) Mamíferos y aves depredados por Tyto alba (Aves: Strigiformes) en zonas urbanas y rurales de Montevideo. Actas de las V Jornadas de Zoología del Uruguay y I Encuentro Nacional de Entomología Médica y Veterinaria, Noviembre 1999, Montevideo, Uruguay. Boletín de la Sociedad Zoológica del Uruguay (2a Epoca) $11: 14$. expansión hacia el norte (Grigera \& Rapoport 1983, de la Sancha et al. 2009). En Uruguay se distribuye en todo el territorio, apareciendo citada en las primeras listas de fauna (Sanborn 1929, Devincenzi 1935, Palerm 1950, Legrand 1959, Tálice 1969, Vaz Ferreira 1969, Del Pino 1988, González 2001, Achaval et al. 2007), lo que se condice con el análisis de idoneidad de hábitat (Fig. 1D). Sin haber sido evaluado su efecto, se considera como plaga para la agricultura (Sanborn 1929, Del Pino 1988). La liebre es además utilizada como especie cinegética y de explotación industrial. La legislación actual no requiere la autorización gubernamental para su caza (MGAP Decreto 161/003). Se ha conformado con $L$. europeus la industria exportadora de carne de caza más importante del país desde principios de los 90 ' y se realiza también exportación de especímenes vivos para cotos de caza (INAC 2010). Uruguay exportó aproximadamente 200.000 liebres por año (Rovere \& Uriarte 2003) durante un período en el cual el mercado europeo para esta especie se encontraba en crecimiento (Silva 2007). Si se consideran las pérdidas industriales por descarte de especímenes, los miles de ejemplares colectados vivos para exportación y por caza deportiva, el total de liebres extraídas anualmente podría superar los 250.000 individuos (INAC 2010, Rovere \& Uriarte 2003).

Jabalí (Sus scrofa Linnaeus, 1758)

Aunque el jabalí fue introducido en el Departamento de Colonia en la década de 1920, existen indicios que indican que anteriormente ya existían en Uruguay poblaciones de cerdos salvajes (Araújo 1900), con los que se reprodujeron (Herrero \& Fernández De Luco 2003). Esta especie se encuentra aún en estado de expansión, ocurriendo periódicamente nuevos registros (Vaz Ferreira 1969, Del Pino 1988, Herrero \& Fernández De Luco 2003, Lombardi et al. 2007). Se han estimado densidades entre 0.33 y 1.17 individuos $\mathrm{km}^{-2}$ (Lombardi et al. 2007). El jabalí presenta una buena idoneidad de hábitat, aunque con variaciones a lo largo del territorio (Fig. 1E). Se alimenta de plantas, raíces, carroña y larvas de insectos (Herrero \& Fernández De Luco 2003, Skewes et al. 2007, Sanguinetti \& Kitzberger 2010). Esta especie ha llamado la atención de las autoridades nacionales debido a sus 
efectos directos sobre actividades productivas, destruyendo plantaciones e incluso depredando sobre las majadas, especialmente de corderos recién nacidos (Vaz Ferreira 1969, Del Pino 1988, Herrero et al. 2006, Vaz Ferreira 1969). Además, ha sido identificada recientemente como vector de Mycobacterium tuberculosis Koch (Naranjo et al. 2008) y es considerada un importante vector de aftosa (e.g., Pech \& McIlroy 1990, Dexter 2003). Se ha propuesto que pudo afectar negativamente a especies nativas por depredación, como las poblaciones silvestres del venado de campo Ozotoceros bezoarticus Linnaeus, 1758 (Pérez Carusi et al. 2009) y carpinchos Hydrochoerus hydrochaeris Linnaeus, 1766 (Lombardi et al. 2007). E1 jabalí es objeto de caza deportiva (Ojasti 1993), desarrollándose incluso jornadas de promoción y competencias de caza de la especie, aunque esta no es considerada una medida de manejo efectiva para controlar esta invasión (Herrero \& Fernández De Luco 2003). Las zonas de caza abarcan principalmente serranías, humedales y bosques galería (Lombardi et al. 2007).

Vizcacha (Lagostomus maximus Desmarest, 1817)

Esta especie fue traída desde Argentina e introducida en Artigas, al Norte de Uruguay, en 1889, calculándose que en 1920 existían unos dos mil especímenes silvestres (Vaz Ferreira 1969). A raíz del daño que ocasionaban, fueron fuertemente perseguidas y erradicadas en 1922, no volviendo a registrarse ejemplares (Tálice 1969, Vaz Ferreira 1969).

Conejo europeo (Oryctolagus cuniculus Linnaeus, 1758)

El conejo europeo fue introducido dando lugar a poblaciones silvestres en las islas oceánicas y del Río de la Plata. Si bien fueron introducidos individuos en el continente en repetidas ocasiones, a diferencia de lo que estaría sucediendo en Argentina y Chile aparentemente no se habrían logrado poblaciones exitosas (Vaz Ferreira 1969, Jaksic \& Soriguer 1981, Jaksic 1986, Bonino \& Gader 1987, Bonino \& Soriguer 2004). En la Isla Verde, la isla oceánica más al este del país, los conejos fueron introducidos en 1907 y alcanzaron altas densidades en la década del $30^{\prime}$, produciendo un efecto importante sobre la comunidad vegetal nativa (Ximénez \& Langguth 2008). La situación es similar en la Isla del Marco, donde fueron abundantes hasta los 60' y luego desaparecieron (Ximénez \& Langguth 2008). En la actualidad, la especie se presume extinguida en las islas donde fuera introducida (Vaz Ferreira 1956, Arechavaleta 1882, Ximénez \& Langguth 2002, Ximénez \& Langguth 2008), aunque esto podría deberse a que sus poblaciones presentan fuertes fluctuaciones de abundancia llevándolas en ciertos períodos a números que podrían causar su extinción (Ximénez \& Langguth 2002). Esta especie se alimenta de pasturas nativas y también consume los cactus introducidos Opuntia aurantiaca Lindley, 1833 (Ximénez \& Langguth 2002) y es depredado por Tyto alba (Claramunt \& González 1999, op. cit.). Los especímenes silvestres pueden ser reservorio de patologías, como el virus de la mixomatosis, que afectan a la cría comercial de conejos (Aparicio et al. 2006).

\section{Ciervo Axis (Axis axis Erxleben, 1777)}

El ciervo Axis fue introducido a comienzos del siglo XX en el Departamento de Colonia con fines cinegéticos (González 1979, Del Pino 1988, González \& Seal 1997) y luego una población fue trasladada al Departamento de Rocha (González \& Seal 1997). Actualmente, se ha expandido a gran parte del país (Del Pino 1988, González 1979), lo que es congruente con su idoneidad de hábitat (Fig. 1F). En el Parque Anchorena en Colonia, localidad donde fuera originalmente introducido, afecta las plantaciones de árboles ornamentales (González \& Seal 1997) pero no existe información sobre su impacto en comunidades nativas. El Axis es objeto de caza deportiva (Ojasti 1993) siendo necesario contar con autorización para realizarla (MGAP Decreto 164/96). La especie está relacionada al ciclo del parásito Echinococcus granulosus (González 1989) y al igual que la mayoría de los artiodáctilos, con la fiebre aftosa (Thomson et al. 2003).

\section{Gamo (Dama dama Linnaeus, 1758)}

La especie fue introducida en el Departamento de Florida y aparentemente no habría sido tan exitoso, ya que se encontraría restringido a 
un área pequeña del centro del país (González 1979, González 2001, Achaval et al. 2007). Este patrón es congruente con la estimación de idoneidad de hábitat (Fig. 1G). Es utilizada para caza deportiva (Ojasti 1993) pero es necesaria autorización para realizarla (MGAP Decreto 164/96). El gamo es también vector de la fiebre aftosa (Thomson et al. 2003).

\section{Cabra doméstica (Capra hircus Linnaeus, 1758)}

Las poblaciones naturalizadas de la cabra doméstica se han establecido en los Departamentos de Lavalleja (Achaval et al. 2007) y Maldonado (RPG 2006 obs. pers). Hasta el momento no existen estudios sobre el potencial impacto e interacciones de la especie en las comunidades nativas. El análisis de idoneidad de hábitat sugiere un potencial para su difusión desde ubicaciones actuales (Fig. 1H). Esta especie también es un posible reservorio de fiebre aftosa (Thomson et al. 2003).

Búfalo acuático (Bubalus bubalis Linnaeus, 1758)

E1 búfalo acuático fue introducido hace aproximadamente veinte años en el norte del país. Las poblaciones naturalizadas están presentes en Artigas y Rivera (González 2001, Achaval et al. 2007). Sin embargo, se desconoce el estado de estas poblaciones y se estima una baja idoneidad de hábitat (Fig. 1I).

Visón americano (Neovison vison Schreber, 1777)

E1 visón americano, $N$. vison, ha sido introducido con fines productivos en Uruguay (DEC. $\mathrm{N}^{0}$ 828/008). Aunque no existen poblaciones silvestres, trabajos de cría piloto, realizados al Sur del país, identificaron que la especie encuentra condiciones ambientales adecuadas para desarrollarse como animal peletero de granja (Otero et al. 2006). Este tipo de experiencias han dado lugar a focos de invasión con especies como la rana toro, Lithobates catesbeianus Shaw, 1802 (Laufer et al. 2009) y la carpa Cyprinus carpio Linnaeus, 1758 (Ares et al. 1991). Notablemente, la estimación de idoneidad sugiere que la especie podría establecerse y dispersarse por la mayor parte del territorio (Fig. 1J).

Ardilla vientre rojo (Callosciurus erythraeus Pallas, 1779)

La estimación de idoneidad de hábitat para C. erythraeus, la ardilla de vientre rojo, es notablemente baja con un par de sitios con relativamente alta probabilidad de establecimiento (Fig. 1K). No obstante, la situación en la zona de Luján, Provincia de Buenos Aires, Argentina, donde la especie ha sido introducida y se expande con éxito (Guichón et al. 2005), sugiere cautela sobre el eventual riesgo de establecimiento de esta ardilla en el Uruguay.

Considerando la superposición de las capas de distribución potencial, encontramos que los mamíferos exóticos considerados presentaron patrones de idoneidad de hábitat no homogéneos, implicando la existencia de regiones del Uruguay en donde la posibilidad de su establecimiento es considerablemente mayor. Específicamente, en el Sur del país se identificaron dos regiones con condiciones excepcionalmente altas para la radicación de especies exóticas en los Departamentos costeros de Colonia y Maldonado (Fig. 1L).

\section{Cambios en la estructura trófica de la biota}

Los patrones de extinción e introducción de especies no fueron homogéneos entre grupos tróficos en Uruguay (Fig. 2), siendo los grandes carnívoros, los más afectados. Desde la llegada de los colonizadores europeos, se han extinguido en el territorio uruguayo: el ciervo de los pantanos (Blastocerus dichotomus Illiger, 1815), el Jaguar (Panthera onca Linnaeus, 1758), el lobo grande de río (Pteronura brasiliensis Gmelin, 1788), el oso hormiguero grande (Myrmecophaga tridactyla Linnaeus, 1758) y el pecarí (Pecari tajacu Linnaeus, 1758). Por otra parte, el puma (Puma concolor Linnaeus, 1771) y el ocelote (Leopardus pardalis Schinz, 1821) cuentan con registros escasos y puntuales en los últimos años por lo que los consideramos extinguidos para este análisis. En este panorama, los carnívoros han perdido un $40 \%$ de las especies nativas (Fig. 2A) y teniendo en cuenta las especies con algún grado de amenaza podrían perder hasta un 
(A)

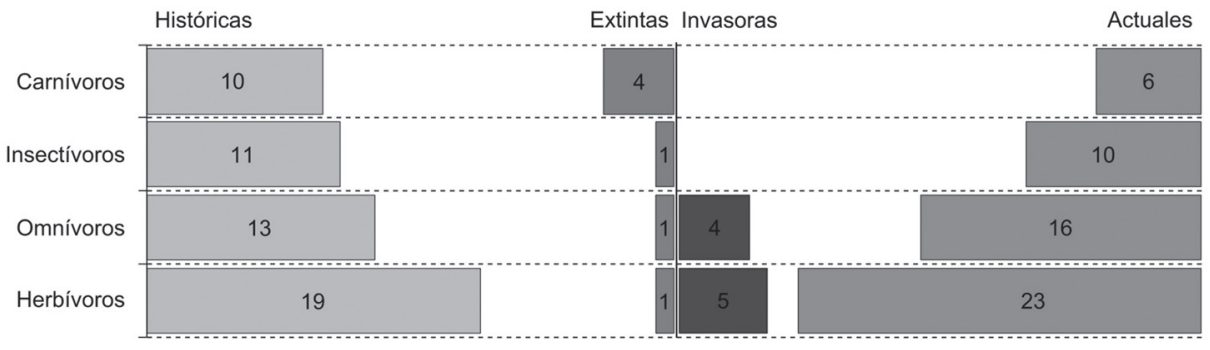

(B)

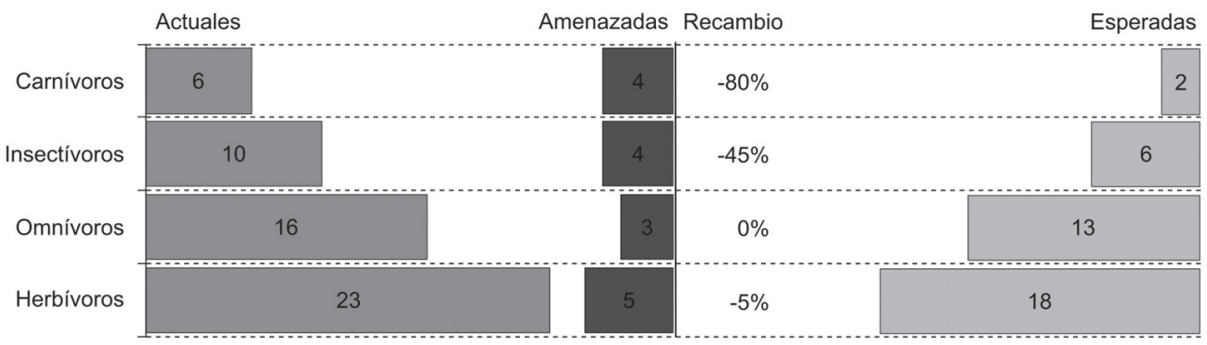

(C)

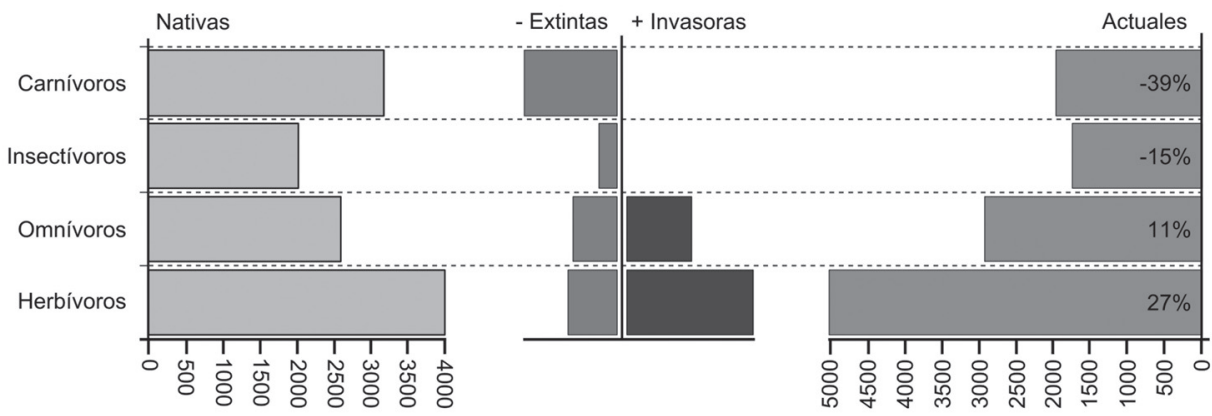

Fig. 2: Cambios en la estructura trófica en la biota de mamíferos del Uruguay debido a procesos de extinción e invasión; (A) cambios en el número de especies debido a las extinciones e invasiones ya ocurridas; (B) cambios esperados, en el número de especies, asumiendo la extinción de las especies amenazadas; (C) cambios esperados en la biomasa de los distintos grupos tróficos.

Changes in trophic structure of mammalian biota as consequence of the ongoing processes of species introduction and extinctions; (A) changes in species number due to extinctions and invasions which have already occurred; (B) expected changes in species number assuming the extinction of endangered species; (C) Expected changes in estimated biomasses for different trophic groups.

$80 \%$ (Fig. 2B). Los insectívoros han perdido un $9 \%$ de especies, que podría llegar al $45 \%$. Por su parte, los omnívoros, serían los menos afectados en pérdida de especies pues el número de invasores es igual al número de especies que podrían llegar a perderse, y los herbívoros, en una situación similar, podrían llegar a perder un $5 \%$ de las especies nativas pre-colonia. Tomando en cuenta introducciones y extinciones, los carnívoros e insectívoros sólo perdieron especies, mientras que los omnívoros y herbívoros en conjunto presentan un saldo neto de ganancia de especies $(=7.9 ; \mathrm{P}$ $<0.005)$. Así mismo, se detectaron diferencias significativas en el tamaño corporal de las especies que componen la biota de mamíferos nativos e introducidos en el Uruguay $\left(\mathrm{F}_{1,38}=\right.$ 4.87 $\mathrm{P}=0.033)$. En este sentido, los mamíferos 
habrían perdido un $39 \%$ de representación de su biomasa y los insectívoros un $15 \%$. Por su parte los omnívoros y herbívoros habrían aumentado sustancialmente sus biomasas en $11 \%$ y $27 \%$ respectivamente (Fig. 2C). Específicamente, los herbívoros invasores presentan mayor tamaño corporal que las especies nativas $\left(\mathrm{F}_{1,22}=12.3 \mathrm{P}=0.002\right)$. Por su parte las especies extintas son las de mayor tamaño en todos los grupos tróficos.

\section{DISCUSIÓN}

A pesar del pequeño tamaño de Uruguay, existe gran variabilidad geográfica en la idoneidad de hábitat para las especies de mamíferos exóticos. Esto es congruente con la existencia de una importante diversidad climática y de biomas en esta restringida región del continente (González \& Martinez-Lanfranco 2010). Dependiendo de la especie y el sitio de introducción, podrían darse escenarios variados en el eventual éxito y desarrollo de los procesos de invasión (Arim et al. 2006). En este sentido, la identificación de regiones de alto riesgo podría ser base para criterios de manejo. Los patrones geográficos en distintos grupos taxonómicos suelen estar correlacionados (Toranza \& Arim 2010), por lo que el patrón aquí reportado para mamíferos podría ser congruente con el eventual riesgo de establecimiento de especies exóticas de otros grupos taxonómicos. Las áreas de Maldonado y Colonia con alta idoneidad para el establecimiento de especies exóticas son también áreas con un importante efecto de alteración antrópica por la expansión urbanística, productiva y turística de la zona costera (Menafra et al. 2006). Este contexto genera una mayor probabilidad de vías y de frecuencia de ingreso de organismos exóticos (Lockwood et al. 2007). El éxito de las invasiones suele estar asociado al número de introducciones realizadas y a la idoneidad de las condiciones locales (Lockwood et al. 2007). Por tanto, el escenario en estos Departamentos sería de particular vulnerabilidad a los procesos de llegada, establecimiento y eventual dispersión de especies exóticas.

Las especies invasoras en Uruguay tienen diferentes atributos ecológicos que las nativas, impactando en la composición de la biota de mamíferos (Mack et al. 2000). Este cambio podría afectar seriamente a la estructura y funcionamiento de los ecosistemas. La riqueza específica, biomasa y distribución de tamaños corporales están siendo impactadas por los procesos de introducción y extinción de especies (Fig. 2). En este proceso se están extirpando especies de las posiciones tróficas superiores, lo que implica una pérdida de diversidad y biomasa de las comunidades nativas, quedando mayormente organismos depredadores de menor tamaño. Contrariamente, la base de las redes tróficas podría estar aumentando en biomasa, riqueza e incluso en tamaño corporal por la introducción de mamíferos exóticos. Patrones de cambio similares ya han sido reportados para sistemas intermareales, pero no en sistemas terrestres (Byrnes et al. 2007). Este proceso es probablemente compartido por otros países de la región como Argentina (Novillo \& Ojeda 2008) y Chile (Jaksic 1998, Jaksic et al. 2002), estableciéndose herbívoros de tamaño mayor y carnívoros de menor porte que las especies nativas. Estas tendencias son congruentes con la hipótesis de "dos caras de una moneda" según la cual los atributos de las especies invasoras son contrarios a los que presentan las especies en peligro o extintas (Jeschke \& Strayer 1998). No obstante, la invasión exitosa por especies de gran tamaño (e.g. Sus scrofa, Axis axis) es contraria a la visión de que éstas son particularmente sensibles a los procesos de cambio ambiental (Pimm 1991, Marquet \& Taper 1998, Burness et al. 2001), aunque si sería el caso en los carnívoros. En este sentido, debe considerarse que la disminución de grandes especies y principalmente en altas posiciones tróficas, suele asociarse a efectos humanos indirectos de deterioro ambiental. En el caso de Uruguay el componente humano directo, a través de la caza e introducción intencional de especies, probablemente ha jugado un papel central. Grandes herbívoros y omnívoros fueron activamente introducidos con fines de caza deportiva, mientras que los carnívoros de gran porte fueron activamente diezmados (Pereira-Garbero \& Sappa, en prensa). En consecuencia, los cambios en grupos tróficos en la biota de mamíferos de Uruguay probablemente representan acciones humanas directas y no una respuesta a cambios en los ecosistemas.

A las acciones directas del hombre puede sumarse un contexto ecológico favorable 
a grandes herbívoros y omnívoros, experimentando un escenario particular de baja depredación y competencia. La acción directa del hombre eliminó a los mamíferos depredadores de gran tamaño, no solo en los pasados siglos (Pereira-Garbero \& Sappa, en prensa) sino con su llegada a América hace milenios (Wroe et al. 2006). También debido a la acción del hombre u otros procesos (Lyons et al. 2004) hace aproximadamente 10 mil años se extinguió una excepcional diversidad de megafauna (Barnosky et al. 2004) mayoritariamente herbívora, con algunos representantes omnívoros (Fariña et al. 1998). Así mismo, los cérvidos nativos fueron diezmados a gran escala en los primeros siglos de colonización del nuevo mundo (de Varnhagen, 1839). De esta forma, podría relacionarse el éxito relativo de los mamíferos invasores, en su mayoría herbívoros y omnívoros, con la extinción y disminución de sus eventuales depredadores y competidores.

Los cambios a escala de la biota de mamíferos para todo el país pueden sobre o subestimar el impacto de estos procesos en las comunidades locales. Es esperable que la extinción de carnívoros mayores a $15 \mathrm{~kg}$, haya impactado en la mayoría de las comunidades locales, ya que el tamaño corporal de los depredadores, determina muy baja mortalidad por depredación en ciertos rangos de tamaño de presas (Sinclair et al. 2003). Estos carnívoros tienen gran capacidad de movimiento, consumen en diversas redes locales para satisfacer sus elevadas demandas energéticas y presentan amplia diversidad trófica (McCann et al. 2005, Arim et al. 2010). Congruentemente, los pocos casos de reintroducción de carnívoros de tamaño intermedio han demostrado afectar varios ecosistemas y no solo los directamente afectados por depredación (Croll et al. 2005, Kurle et al. 2008). De forma similar, la remoción de grandes herbívoros ha demostrado afectar distintos tipos de comunidades, cambiando su diversidad y funcionamiento (Hopcraft et al. 2009).

Es esperable que la extinción de la megafauna, hace pocos miles de años, haya afectado a gran parte del territorio. La reciente introducción de herbívoros de gran tamaño, como el ganado vacuno, equinos, suinos y ovinos, aunque mucho menores que la megafauna extinta, habría incrementado la riqueza de este grupo en algunas zonas. Por otra parte, la distribución real de la mayoría de las especies introducidas es desconocida, no pudiéndose calibrar el impacto de estos procesos en las comunidades locales. No obstante, la estimación del impacto de las introducciones en general requiere, por un lado, de la realización de estudios de campo a escalas espaciales mucho más reducidas, y por otro, de la integración de datos del proceso de invasión y extinción a nivel regional. Las hipótesis de idoneidad ambiental aquí propuestas podrían ser un punto de partida para evaluar el establecimiento y avance de especies exóticas en el territorio, su variabilidad en abundancia y el impacto en las comunidades locales.

Frecuentemente la introducción de especies exóticas es sustentada por enunciados con poco o ningún respaldo científico. Las condiciones ambientales supuestamente adversas, la ausencia de especies similares, disponibilidad de nichos vacíos, la imposibilidad de escapes o reproducción en vida libre, han sido esgrimidas para permitir el ingreso de especies exóticas. El principal problema de estas consideraciones es que no se desprenden de estudios adecuadamente sustentados en experimentos, análisis comparativos o las herramientas y marcos teóricos disponibles. La estimación de idoneidad ambiental para el visón americano discrepa fuertemente con la supuesta ausencia de condiciones para su establecimiento. Por ser un carnívoro agresivo, esta especie sería una amenaza mayor para la biodiversidad de micromamíferos, reptiles e invertebrados (Ahola et al. 2006). La suposición de "hábitat no apto", fue también utilizada en Uruguay para la introducción de rana toro (Lithobates catesbeianus). Esta especie también muestra zonas de alta idoneidad climática, donde se han reportado recientemente tres poblaciones silvestres (Laufer et al. 2008, Laufer et al. 2009). La decisión de introducción de especies con fines productivos, ornamentales, de caza o mascotas debería basarse en un balance bien sustentado en los eventuales costos y beneficios de las iniciativas. Es imperioso complementar los aportes aquí introducidos con estudios experimentales y de campo, para contar con un marco de referencia para el desarrollo de estas políticas.

El establecimiento de especies exóticas es un proceso que está ocurriendo a escala 
global involucrando todos los grupos taxonómicos (Jaksic et al. 2002, Ricciardi 2007). Un porcentaje importante de los mamíferos exóticos se han establecido en Sudamérica (Novillo \& Ojeda 2008) y cinco de las 14 especies de mamíferos catalogadas como las más dañinas, se encuentran actualmente en Uruguay (Lowe et al. 2004). Es fundamental en este contexto avanzar en la comprensión de la historia natural de las especies exóticas presentes en la región y en sus potenciales impactos en las comunidades nativas. En este sentido resulta prioritario conocer la posibilidad de avance de las diferentes especies a nivel regional. El presente trabajo intentó avanzar en esta línea catalogando a las especies introducidas en Uruguay, presentando su estado actual de establecimiento, atributos biológicos y estimando la idoneidad ambiental para cada una de ellas. Debe destacarse que si bien sólo se utilizaron variables climáticas en la estimación de estas idoneidades, estas suelen estar correlacionadas con otras variables ambientales que potencialmente afectan la distribución de las especies (Elith \& Leathwick 2009). Así mismo, hemos puesto el foco en la importancia de considerar simultáneamente los procesos de incorporación y extinción de especies, los cuales pueden o no estar mutuamente relacionados (Jeschke \& Strayer 2008), pero son y han sido los determinantes de la composición de biotas en todo el planeta.

En las últimas décadas, en varios países se ha establecido una perspectiva proactiva hacia las especies invasoras (Shine et al. 2000), implementándose estrategias para reducir el riesgo de establecimiento de especies exóticas con fines productivos $\mathrm{u}$ ornamentales y el de nuevas introducciones accidentales (IUCN/ ISSC 2000, Lowe et al. 2004, Lodge et al. 2006, Pyke et al. 2008). En este sentido Uruguay debería seguir esta línea de acción, tomando medidas para evitar el ingreso de especies exóticas, y para combatir las ya introducidas en base a experiencias internacionales, estudios de campo y un marco teórico adecuado.

AGRADECIMIENTOS: Este trabajo fue financiado por el proyecto PDT $71 / 10$ para MA. MA agradece el soporte de FONDAP-FONDECYT 1501-0001. Este es un trabajo póstumo de Federico Achaval Elena. La excepcional dedicación de este autor al relevamiento de la biodiversidad de vertebrados da buena cuenta del conocimiento actual de este grupo en el Uruguay. A Federico Achaval Coppes por su apoyo. Agradecemos también a los revisores anónimos, quienes realizaron aportes sustanciales a la mejora del trabajo original.

\section{LITERATURA CITADA}

ACHAVAL F, M CLARA \& A OLMOS (2007) Guía fotográfica de los mamíferos de Uruguay. Segunda edición. Zonalibro Industria Gráfica, Montevideo.

AHOLA M, M NORDSTROM, PB BANKS, N LAANETU \& E KORPIMÄKI (2006) Alien mink predation induces prolonged declines in archipelago amphibians. Proceedings of the Royal Society of London B 1591: 1261-1265.

ALTIERI AH, BK VAN WESENBEECK, MD BERTNESS \& BR SILLIMAN (2010) Facilitation cascade drives positive relationship between native biodiversity and invasion success. Ecology 91: 1269-1275.

ALVAREZ-ROMERO JG, RA MEDELLÍN, A OLIVERAS DE ITA, H GÓMEZ DE SILVA \& O SÁNCHEZ (2008) Animales exóticos en México: Una amenaza para la biodiversidad. CONABIO, Instituto de Ecología UNAM, SEMARNAT, México D.F

ANDERSON CB, GM PASTUR, MV LENCINAS, PK WALLEM, MC MOORMAN \& AD ROSEMOND (2009) Do introduced North American beavers Castor canadensis engineer differently in southern South America? An overview with implications for restoration. Mammal Review 39: 33-52.

APARICIO JP, HG SOLARI \& NA BONINO (2006) Perspectivas teóricas sobre la dinámica de la mixomatosis con aplicaciones en control biológico. Ecología Austral 16: 15-28.

APLIN OV (1894) Field notes on the mammals of Uruguay. Proceedings of the Zoological Society of London 1894: 297-315.

ARAÚJO O (1900) Diccionario geográfico de Uruguay. Imprenta artística de Dornaleche y Reyes, Montevideo, Uruguay.

ARECHAVAlETA J (1882) Reino animal. Álbum de la República Oriental del Uruguay compuesto para la Exposición Continental de Buenos Aires. Imprenta de Rius y Becchi, Montevideo, Uruguay.

ARES L, COLLAZO D \& GARCÍA C (1991) Presencia de Cyprinus carpio (Linnaeus, 1758) (Osteichthyes, Cypriniformes) en costas uruguayas del Río de la Plata. Boletín de la Sociedad Zoológica del Uruguay, Montevideo, Uruguay (2) 6:54-56

ARIM M, SR ABADES, G LAUFER, M LOUREIRO \& PA MARQUET (2010) Food web structure and body size: trophic position and resource acquisition. Oikos 119: 147-153.

ARIM M, SR ABADES, PE NEILL, M LIMA \& PA MARQUET (2006) Spread dynamics of invasive species. Proceedings of the National Academy of Sciences 103: 374-378.

ARISMENDI I, P SZEJNER, A LARA, \& ME GONZÁLEZ (2008) Impacto del castor en la estructura de bosques ribereños de Nothofagus pumilio en Tierra del Fuego, Chile. Bosque 29: 146-154.

BAILEY JK \& JA SCHWEITZER (2009) The role of plant resistance and tolerance to herbivory in mediating the effects of introduced herbivores. Biological Invasions, 12: 337-351.

BALDINI A, J OLTREMARI \& M RAMÍREZ (2008) Impacto del castor (Castor canadensis, Rodentia) 
en bosques de lenga (Nothofagus pumilio) de Tierra del Fuego, Chile. Bosque 29: 162-169.

BARNOSKY AD, PL KOCH, RS FERANEC, SL WING \& AB SHABEL (2004) Assessing the causes of late Pleistocene extinctions on the continents. Science 306: 70-5.

BONINO NA \& R GADER (1987) Expansión del conejo silvestre europeo (Orictolagus cuniculus) en la República Argentina y perspectivas futuras. Anales del Museo de Historia Natural, Valparaíso (Chile) 18: 157-162.

BONINO NA \& RC SORIGUER (2004) Distribución actual y dispersión del conejo europeo (Oryctolagus cuniculus) en Mendoza (Argentina). Mastozoología Neotropical 11: 237-241.

BROWN JH, JF GILLOOLY, AP ALLEN, VM SAVAGE \& GB WEST (2004) Toward a metabolic theory of ecology. Ecology 85: 1771-1789.

BROWN JH \& DF SAX (2004) An essay on some topics concerning invasive species. Austral Ecology 29: 530-536.

BURNESS GP, J DIAMOND \& T FLANNERY (2001) Dinosaurs, dragons, and dwarfs: the evolution of maximal body size. Proceedings of the National Academy of Sciences USA 98: 14518-14523.

BYRNES JE, PL REYNOLDS \& JJ STACHOWICZ (2007) Invasions and extinctions reshape coastal marine food webs. PLoS ONE 2: e295.

CARBONE C \& JL GITTLEMAN (2002) A common rule for the scaling of carnivore density. Science 295: 2273-2276.

CARBONE C, JM ROWCLIFFE, G COWLISHAW \& NJB ISAAC (2007) The scaling of abundance in consumers and their resources: implications for the energy equivalence rule. American Naturalist 170: 479-484.

CARLSSON NO, O SARNELLE\& DL STRAYER (2009) Native predators and exotic prey -an acquired taste? Frontiers in Ecology and the Environment 7: 525-532.

CHAPIN FS, ES ZAVALETA, VT EVINER, RL NAYLOR, PM VITOUSEK et al. (2000) Consequences of changing biodiversity. Nature 405: 234-242.

CONTI DÍAZ IA (2001) Enfermedades emergentes y reemergentes en Uruguay. Revista Médica del Uruguay 17: 180-199.

CROLL DA, JL MARON, JA ESTES, EM DANNER \& GV BYRD (2005) Introduced predators transform subarctic islands from grassland to tundra. Science 307: 1959-1961.

DAMUTH J (1981) Population density and body size in mammals. Nature 290: 699-700.

DAVIS NE, DM FORSYTH \& G COULSON (2010) Facilitative interactions between an exotic mammal and native and exotic plants: hog deer (Axis porcinus) as seed dispersers in southeastern Australia. Biological Invasions 12: 10791092.

DE LA SANCHA N, H MANTILLA-MELUK, F RAMIREZ, P PEREZ, N MUJICA, A TROCHE \& M GIMENEZ (2009) Mammalia, Lagomorpha, Leporidae, Lepus europaeus, Pallas, 1778: Distribution extension, first confirmed record for Paraguay. Check List, 5(3): 428-432.

DEL PINO C (1988). Mamíferos del Uruguay, foráneos integrados a nuestra fauna. Almanaque del Banco de Seguros del Estado, Montevideo, Uruguay 71: 260-262.

DE VARNHAGEN FA (1839) Diario da navegação da armada que foi á terra do Brasil em 1530, escripto por seu irmão Pero Lopes de Souza. Typographia da Sociedade propagadora Dos Conhecimentos Uteis, Lisboa.

DEVINCENZI GJ (1935) Mamíferos del Uruguay. Anales del Museo de Historia Natural de Montevideo, Uruguay (2) 4(10): 1-96.

DEXTER N (2003) Stochastic models of foot and mouth disease in feral pigs in the Australian semi-arid rangelands. Journal of Applied Ecology 40: 293306.

DIDHAM RK, JM TYLIANAKIS, NJ GEMMELL, TA RAND \& RM EWERS (2007) Interactive effects of habitat modification and species invasion on native species decline. Trends in Ecology and Evolution 22: 489-496.

D'ANTONIO CM, LA MEYERSON \& J DENSLOW (2001) Research Priorities related to invasive exotic species. In: Soule ME \& GH Orians (eds) Conservation Biology Research Priorities for the Next Decade: 59-80. DC Island Press, Washington.

ELITH J \& CH GRAHAM (2009) Do they? How do they? WHY do they differ? On finding reasons for differing performances of species distribution models. Ecography 32: 66-77.

ELITH J \& JR LEATHWICK (2009) Species distribution models: Ecological explanation and prediction across space and time. Annual Review of Ecology, Evolution, and Systematics 40: 677-697.

FERNÁNDEZ MA, SD BLUM, S REICHLE, B HOLZMAN \& H HAMILTON (2009) Locality uncertainty and the differential performance of four common niche-based modeling techniques. Biodiversity Informatics 6: 36-52.

FICETOLA GF, W THUILLER \& C MIAUD (2007) Prediction and validation of the potential global distribution of a problematic alien invasive species - the American bullfrog. Diversity and Distributions, 13: 476-485.

FIGUEIRA JH (1894) Contribución al conocimiento de la Fauna Uruguaya. Enumeración de Mamíferos. Anales del Museo Nacional de Montevideo, Uruguay (1)2(5): 187-217.

FRANKLIN J (2010) Mapping species distribution: Spatial inference and prediction. Cambridge University Press, Cambridge.

GALETTI M, RS BOVENDORP, RF FADINI, COA GUSSONI, M RODRIGUES, AD ALVAREZ, PR GUIMARAES \& K ALVES (2009) Hyper abundant mesopredators and bird extinction in an Atlantic forest island Bird survey. Zoologia 26: 288-298.

GONZÁLEZ EM (2001) Guía de campo de los Mamíferos de Uruguay. Introducción al Estudio de los Mamíferos. Vida Silvestre. Montevideo, Uruguay.

GONZÁLEZ E M \& JA MARTÍNEZ-LANFRANCO (2010) Mamíferos de Uruguay. Guía de campo e introducción a su estudio y conservación. Banda Oriental, MNHN y Vida Silvestre Uruguay, Montevideo.

GONZÁLEZ JC (1979) Ciervos autóctonos y exóticos en el Uruguay. Almanaque del Banco de Seguros del Estado, Montevideo, Uruguay 62: 219-223.

GONZÁLEZ JC (1989) Algunas enfermedades transmisibles de los animales al hombre en el Uruguay. Zoonosis. Almanaque del Banco de Seguros del Estado, Montevideo, Uruguay 71: 200-203.

GONZÁLEZ S \& US SEAL (1997) El manejo del ciervo axis (Cervus axis) en la residencia presidencial de Colonia-Uruguay. IUCN/SSC Conservation 
Breeding Specialist Group, Apple Valley, Minnesota, Estados Unidos.

GOTELLI NJ (2008) A primer of ecology. Cuarta edición. Sinauer Associates Inc., Sunderland, Massachusetts.

GRAHAM CH, J ELITH, RJ HIJMANS, A GUISAN, AT PETERSON et al. (2008) The influence of spatial errors in species occurrence data used in distribution models. Journal of Applied Ecology 45: 239-247.

GRIGERA DE \& EH RAPOPORT (1983) Status and distribution of the european hare in south america. Journal of Mammalogy 64: 163-166.

GUICHÓN ML, M BELLO \& L FASOLA (2005) Expansión poblacional de una especie introducida en la argentina: la ardilla de vientre rojo Callosciurus erytrhaeus. Mastozoologia Neotropical 12: 189-197.

HASTINGS A, JE BYERS, JA CROOKS, K CUDDINGTON, CG JONES, JG LAMBRINOS, TS TALLEY \& WG WILSON (2007) Ecosystem engineering in space and time. Ecology letters 10: $153-64$.

HERRERO J \& D FERNÁNDEZ DE LUCO (2003) Wild boars (Sus scrofa) in Uruguay: scavengers or predators? Mammalia 67: 485-491.

HERRERO J, A GARCÍA-SERRANO, S COUTO, VM ORTUÑ̃O \& R GARCÍA-GONZÁLEZ (2006) Diet of wild boar Sus scrofa L. and crop damage in an intensive agroecosystem. European Journal of Wildlife Research 52: 245-250.

HIJMANS RJ, SE CAMERON, JL PARRA, PG JONES \& A JARVIS (2005) Very high resolution interpolated climate surfaces for global land areas. International Journal of Climatology 25: 1965-1978.

HOPCRAFT JGC, H OLFF \& ARE SINCLAIR (2009) Herbivores, resources and risks: alternating regulation along primary environmental gradients in savannas. Trends in Ecology \& Evolution 25: 119-128.

HULME PE (2009) Trade, transport and trouble: managing invasive species pathways in an era of globalization. Journal of Applied Ecology 46: 1018.

INAC (2010) Anuario estadístico 2009. Parte 4: Exportación. Montevideo.

IRIARTE JA, GA LOBOS \& FM JAKSIC (2005) Invasive vertebrate species in Chile and their control and monitoring by governmental agencies. Revista Chilena de Historia Natural 78: 143-154.

IUCN/ISSC (2000) IUCN guidelines for the prevention of biodiversity loss caused by alien invasive species. IUCN, Gland, Switzerland.

IUCN (2010) IUCN Red list of threatened species. International Union for Conservation of Nature and Natural Resources, Versión 2010.4. URL: http://www.iucnredlist.org (accedido Febrero 22, 2011)

JAKSIC FM (1986) Predation upon small mammals in shrublands and grasslands of South America: Ecological correlates and presumable consequences. Revista Chilena de Historia Natural 59: 209-221.

JAKSIC FM (1998) Vertebrate invaders and their ecological impacts in Chile. Biodiversity and Conservation 7: 1427-1445.

JAKSIC FM, JA IRIARTE, JE JIMÉNEZ \& DR MARTINEZ (2002) Invaders without frontiers:
Cross-border invasions of exotic mammals. Biological Invasions 4: 157-173.

JAKSIC FM \& RC SORIGUER (1981) Predation upon the European rabbit (Oryctolagus cuniculus) in Mediterranean habitats of Chile and Spain: A comparative analysis. Journal of Animal Ecology 50: 269-281.

JAKSIC FM \& JL YANEZ (1983) Rabbit and fox introductions in Tierra del Fuego: history and assessment of the attempts at biological control of the rabbit infestation. Biological Conservation 26: 367-374

JESCHKE JM \& DL STRAYER (2008) Are threat status and invasion success two sides of the same coin? Ecography 31: 124-130.

JONES CG, JH LAWTON \& M SHACHAK (1994) Organisms as ecosystem engineers. Oikos 69 : 373-386.

KURLE CM, DA CROLL \& BR TERSHY (2008) Introduced rats indirectly change marine rocky intertidal communities from algae- to invertebrate-dominated. Proceedings of the National Academy of Sciences USA 105: 3800 3804.

LA PIERRE KJ, WS HARPOLE \& KN SUDING (2010) Strong feeding preference of an exotic generalist herbivore for an exotic forb: a case of invasional antagonism. Biological Invasions 12: 3025-3031.

LAUFER G, M ARIM \& M LOUREIRO (2009) Informe de dos nuevas poblaciones invasoras de rana toro en Uruguay, presentado a DINAMA y DINARA. Proyecto Invasiones Acuáticas en Uruguay, PDT 71/10, Montevideo.

LAUFER G, A CANAVERO, D NÚÑEZ \& R MANEYRO (2008) Bullfrog (Lithobates catesbeianus) invasion in Uruguay. Biological Invasions 10: 1183-1189.

LEGRAND CD (1959) Comunidades psamófilas de la región de Carrasco (Uruguay). Anales del Museo de Historia Natural Montevideo, Uruguay (2)6(7): $1-65$.

LOCKWOOD JL, MF HOOPES \& MP MARCHETTI (2007) Invasion Ecology. Blackwell Publishing, Malden, MA.

LODGE DM, S WILLIAMS, HJ MACISSAC, KR HAYES, B LEUNG, S REICHARD, et al. (2006) Biological Invasions: recommendations for U.S. policy and management. Ecological Applications 16: 20352054.

LOMBARDI R, R BERRINI, F ACHAVAL \& C WAYSON (2007) El jabalí en Uruguay. Centro Interdisciplinario para el Desarrollo, Montevideo.

LOUDON ASI \& JD CURLEWIS (1988) Cycles of antler and testicular growth in an aseasonal tropical deer (Axis axis). Journal of reproduction and fertility 83: 729-38.

LOWE S, M BROWNE, S BOUDJELAS \& M DE POORTER (2004) 100 de las especies exóticas invasoras más dañinas del mundo. Una selección del Global Invasive Species Database. SGES, SSC, IUCN. Auckland, Nueva Zelandia.

LYONS SK, FA SMITH \& JH BROWN (2004) Of mice, mastodons and men: Human-mediated extinctions on four continents. Evolutionary Ecology Research 6: 339-358.

MACK RN, D SIMBERLOFF, WM LONSDALE, H EVANS, M CLOUT \& FA BAZZAZ (2000) Biotic invasions: Causes, epidemiology, global consequences and control. Ecological Applications 10: 689-710. 
MARQUET PA \& H COFRÉ (1999) Large temporal and spatial scales in the structure of mammalian assemblages in South America: A macroecological approach. Oikos 85: 299-309.

MARQUET PA, FA LABRA \& BA MAURER (2004) Metabolic ecology: Linking individuals to ecosystems. Ecology 85: 1794-1796.

MARQUET PA \& ML TAPER (1998) On size and area: Patterns of mammalian body size extremes across landmasses. Evolutionary Ecology 12: 127-139.

MATEO R, AM FELICÍSIMO \& J MUÑOZ (2011) Modelos de distribución de especies: Una revisión sintética. Revista Chilena de Historia Natural 84: 217-240.

MAY RM, JH LAWTON \& NE STORK (1995) Assesing extinction rates. In: Lawton JH \& RM May (eds) Extinction rates: 1-24. Oxford University Press, Oxford, Reino Unido.

MCCANN KS, JB RASMUSSEN \& J UMBANHOWAR (2005) The dynamics of spatially coupled food webs. Ecology Letters 8: 513-523.

MENAFRA R, L RODRÍGUEZ-GALLEGO, F SCARABINO \& D CONDE (eds) (2006) Bases para la conservación y manejo de la costa uruguaya. Vida Silvestre, Montevideo, Uruguay.

NARANJO V, C GORTAZAR, J VICENTE \& J DE LA FUENTE (2008) Evidence of the role of European wild boar as a reservoir of Mycobacterium tuberculosis complex. Veterinary Microbiology 127: $1-9$.

NAVEH Z, AS LIEBERMAN, FO SARMIENTO, CM GHERSA \& RJC LEÓN (2001) Ecología de paisajes: Teoría y aplicación. Editorial Facultad de Agronomía, Universidad de Buenos Aires, Buenos Aires.

NEILL PE \& M ARIM (2011) Human health link to invasive species. In: Nriagu JO (ed) Encyclopedia of Environmental Health, volume 3: 116-123.

NOVILLO A \& RA OJEDA (2008) The exotic mammals of Argentina. Biological Invasions 10: 1333-1344.

NUÑEZ MA, MA RELVA \& D SIMBERLOFF (2008) Enemy release or invasional meltdown? Deer preference for exotic and native trees on Isla Victoria, Argentina. Austral Ecology 33: 317-323.

OJASTI J (1993) Utilización de la fauna silvestre en América Latina: situación y perspectivas para un manejo sostenible. Guía FAO Conservación 25. FAO, Roma.

OTERO EA, FA HOZBOR, S TORRES, A ECHENIQUE, C CABRERA \& G CAPRA (2006) Guía práctica para la producción del visón (Mustela vison) en Uruguay. INIA, Montevideo.

O'DOWD DJ, PT GREEN \& PS LAKE (2003) Invasional "meltdown" on an oceanic island. Ecology Letters 6: 812-817.

O'REILLY-WAPSTRA JM \& P COWAN (2009) Native plant/herbivore interactions as determinants of the ecological and evolutionary effects of invasive mammalian herbivores: the case of the common brushtail possum. Biological Invasions 12: 373387.

PALERM E (1950) Investigaciones zoogeográficas. Algunas observaciones acerca de los mamíferos de la zona del noroeste del Departamento de Lavalleja. Revista Uruguaya de Geografía. Montevideo, Uruguay 1: 1-85.

PECH RP \& JC MCILROY (1990) A model of the velocity of advance of foot and mouth disease in feral pigs. Journal of Applied Ecology 27: 635-650.
PEREIRA-GARBERO R \& A SAPPA (en prensa) Historia del Jaguar en Uruguay y la Banda Oriental. En: Medellín RA, A de la Torre, C Chávez, H Zarza \& G Ceballos (eds). El jaguar en el siglo XXI: la perspectiva continental. Fondo de Cultura Económica, México D.F.

PETERSON AT \& CR ROBINS (2003) Using ecologicalniche modeling to predict barred owl invasions with implications for spotted owl conservation. Conservation Biology 17: 1161-1165.

PHILLIPS SJ, RP ANDERSON \& RE SCHAPIRE (2006) Maximum entropy modeling of species geographic distributions. Ecological Modelling 190: 231-259.

PHILLIPS SJ \& M DUDIK (2008) Modeling of species distributions with Maxent: new extensions and a comprehensive evaluation. Ecography 31: 161-175.

PIMM SL (1991) The balance of nature? Ecological issues in the conservation of species in communities. University of Chicago Press, Chicago.

PIMM SL, MP MOULTON \& LJ JUSTICE (1995). Bird extinctions in the central pacific. In: JH Lawton \& RM May (eds) Extinction rates: 75-87. Oxford University Press, Oxford, Reino Unido.

PYKE CR, R THOMAS, RD PORTER, JJ HELLMANN, JS DUKES, DM LODGE \& G CHAVARRIA (2008) Current practices and future opportunities for policy on climate change and invasive species. Conservation biology 22: 585-92.

PÉREZ CARUSI LC, MS BEADE, F MIÑARRO, AR VILA, M GIMÉNEZ-DIXON \& D BILENCA (2009) Relaciones espaciales y numéricas entre venados de las pampas (Ozotoceros bezoarticus celer) y chanchos cimarrones (Sus scrofa) en el Refugio de Vida Silvestre Bahía Samborombón, Argentina. Ecología Austral 19: 63-71.

QUIROZ CL, A PAUCHARD, LA CAVIERES \& CB ANDERSON (2009) Análisis cuantitativo de la investigación en invasiones biológicas en Chile: tendencias y desafíos. Revista Chilena de Historia Natural 82: 497-505.

RAMAKRISHNAN U, RG COSS \& NW PELKEY (1999) Tiger decline caused by the reduction of large ungulate prey: evidence from a study of leopard diets in southern India. Biological Conservation 89: $113-120$

RAMAN TRS (1997) Factors influencing seasonal and monthly changes in the group size of chital or axis deer in southern India. Journal of Biosciences 22: 203-218.

RELVA MA, MA NUÑEZ \& D SIMBERLOFF (2009) Introduced deer reduce native plant cover and facilitate invasion of non-native tree species: evidence for invasional meltdown. Biological Invasions 12: 303-311.

RICCIARDI A (2007) Are modern biological invasions an unprecedented form of global change? Conservation Biology 21: 329-36.

ROCHA RG, E FERREIRA, YLR LEITE, C FONSECA \& LP COSTA (2011) Small mammals in the diet of barn owls, Tyto alba (Aves: Strigiformes) along the mid-Araguaia river in central Brazil. Zoologia (Curitiba) 28: 709-716.

ROONEY N, KS MCCANN \& JC MOORE (2008) A landscape theory for food web architecture. Ecology Letters 11: 867-881.

ROVERE G \& G URIARTE (2003) Recursos zoogenéticos. Informe Uruguay. MGAP, Montevideo. 
SALO P, E KORPIMÄKI, PB BANKS, M NORDSTRÖM \& CR DICKMAN (2007) Alien predators are more dangerous than native predators to prey populations. Proceedings of the Real Society B 274: $1237-1243$.

SANBORN CC (1929) The land mammals of Uruguay. Field Museum of Natural History, Zoological Series 17(4): 145-165.

SANGUINETTI J \& T KITZBERGER (2010) Factors controlling seed predation by rodents and nonnative Sus scrofa in Araucaria araucana forests: potential effects on seedling establishment. Biological Invasions 12: 689-706.

SCHMITT SM, SD FITZGERALD, TM COOLEY, CS BRUNING-FANN, L SULLIVAN et al. (1997) Bovine tuberculosis in free-ranging white-tailed deer from Michigan. Journal of Wildlife Diseases 33: 749-758.

SCHEIBLER DR \& AU CHRISTOFF (2007) Habitat associations of small mammals in southern Brazil and use of regurgitated pellets of birds of prey for inventorying a local fauna. Brazilian Journal of Biology 67: 619-25.

SHEPHERD JD \& RS DITGEN (2005) Human use and small mammal communities of Araucaria forests in Neuquén, Argentina. Mastozoología Neotropical 12: 217-226.

SHINE C, N WILLIAMS \& L GÜNDLING (2000) A guide to design legal and institutional frameworks on alien invasive species. Environmental Policy and Law Paper 40. IUCN, Gland, Switzerland.

SILVA AM (2007) Estudio de mercado de la carne de liebre. Universidad de Chile. Santiago.

SIMBERLOFF D \& B VON HOLLE (1999) Positive interactions of nonindigenous species: invasional meltdown? Biological Invasions 1: 21-32.

SINCLAIR ARE, S MDUMA \& JS BRASHARES (2003) Patterns of predation in a diverse predator-prey system. Nature 425: 288-290.

SKEWES O, R RODRIGUEZ \& FM JAKSIC (2007) Ecología trófica del jabalí europeo (Sus scrofa) silvestre en Chile. Revista Chilena de Historia Natural 80: 295-307.

TÁlICE RV (1969) Mamíferos autóctonos. Nuestra Tierra 5. Montevideo, Uruguay.

THOMSON GR, W VOSLOO \& ADS BASTOS (2003) Foot and mouth disease in wildlife. Virus research 91: $145-61$

TORANZA C \& M ARIM (2010) Cross-taxon congruence and environmental conditions. BMC Ecology 10: 18.

VAZ FERREIRA R (1950) Observaciones sobre la Isla de Lobos. Revista de la Facultad de Humanidades y Ciencias, Montevideo, Uruguay 5: 145-175.
VAZ FERREIRA R (1956) Características generales de las islas uruguayas habitadas por lobos marinos. SOYP Trabajos sobre Islas de Lobos y Lobos marinos, Montevideo, Uruguay 1: 1-23.

VAZ FERREIRA R (1969) Fauna: Conservación y Recursos. Nuestra Tierra 45, Montevideo, Uruguay.

VITOUSEK PM, HA MOONEY, J LUBCHENCO \& JM MELILLO (1997) Human domination of earth's ecosystems. Science 277: 494-499.

WALLEM PK, CG JONES, PA MARQUET \& FM JAKSIC (2007) Identificación de los mecanismos subyacentes a la invasión de Castor canadensis (Rodentia) en el archipiélago de Tierra del Fuego, Chile. Revista Chilena de Historia Natural 80: 309326.

WARDLE DA, PJ BELLINGHAM, T FUKAMI \& CPH MULDER (2007) Promotion of ecosystem carbon sequestration by invasive predators. Biology Letters 3: 479-82.

WEBB SD (2006) The great American biotic interchange: Patterns and processes. Annals of the Missouri Botanical Garden 93: 245-257.

WESTPHAL MI, M BROWNE, K MACKINNON \& I NOBLE (2008) Ecological and evolutionary consequences of biotic homogenization. Biological Invasions 10: 391-398.

WILSON JRU, EE DORMONTT, PJ PRENTIS, AJ LOWE \& DM RICHARDSON (2009) Biogeographic concepts define invasion biology. Trends in Ecology and Evolution 24: 586.

WROE S, J FIELD \& DK GRAYSON (2006) Megafaunal extinction: Climate, humans and assumptions. Trends in Ecology and Evolution 21: 61-62.

WRIGHT JP, CG JONES \& AS FLECKER (2002) An ecosystem engineer, the beaver, increases species richness at the landscape scale. Oecologia 132: $96-101$.

XIMÉNEZ A, A LANGGUTH \& R PRADERI (1972) Lista sistemática de los mamíferos del Uruguay. Anales del Museo Nacional de Historia Natural de Montevideo, Uruguay (2)7(5):1-49

XIMÉNEZ I \& E LANGGUTH (2002) Isla de Lobos. Graphis, Montevideo.

XIMÉNEZ I \& E LANGGUTH (2008) Islas y cabos atlánticos del Uruguay. Zonalibro, Montevideo.

ZAMORA J \& S RIEDEMANN (1999) Animales silvestres como reservorios de leptospirosis en Chile: Una revisión de los estudios efectuados en el país. Archivos de medicina veterinaria 31: 151156. 
